\title{
Inflationary trispectrum for models with large non-Gaussianities
}

\author{
Min-xin Huang and Gary Shiu \\ Department of Physics, University of Wisconsin, Madison, Wisconsin 53706, USA
}

(Received 1 November 2006; published 29 December 2006)

\begin{abstract}
We compute the leading order contribution to the four-point function of the primordial curvature perturbation in a class of single field models where the inflationary Lagrangian is a general function of the inflaton and its first derivative. This class of models includes string motivated inflationary models such as DBI inflation. We find that the trispectrum for some range of parameters could potentially be observed in future experiments. Moreover, the trispectrum can distinguish DBI inflation from other inflation models with large non-Gaussianities which typically have a similar bispectrum. We also derive a set of consistency conditions for $n$-point functions of the primordial curvature perturbation in single field inflation, generalizing Maldacena's result for 3-point functions.
\end{abstract}

DOI: 10.1103/PhysRevD.74.121301

PACS numbers: $98.80 . \mathrm{Cq}, 11.25 . \mathrm{Wx}$

The Cosmic Microwave Background (CMB) provides us with a remarkably detailed snapshot of the early universe. The vast structure that we see today is consistent at present with the simple picture of an almost scale invariant, Gaussian primordial density perturbation generated by inflation. Precision measurements of any small deviation from this simple picture contain valuable information that could constrain cosmological models.

In the case of single field inflation, the non-Gaussian perturbation is governed by the $n$-point functions $(n \geq 3)$ of the curvature perturbation $\zeta$. The leading non-Gaussian features are known as the bispectrum (three-point function) and trispectrum (four-point function), with their sizes conventionally denoted as $f_{N L}, \tau_{N L}$ respectively. The current experimental bound for the bispectrum from WMAP3 is $-54<f_{N L}<114$ [1]. Although the experimental bound for the trispectrum is rather weak at the moment $\left|\tau_{N L}\right|<$ $10^{8}$ [2], the next generation of experiments such as PLANCK will increase the sensitivity to about $\left|\tau_{N L}\right| \sim$ 560 [3]. See also [4,5] for discussions of the issue. The CMB bispectrum and trispectrum, if observed, could provide strong constraints on inflationary models. In particular, the shape of the trispectrum contains even more information about the inflationary dynamics than the bispectrum because of the richer varieties of momentum dependence it can encode. As we shall see, in some string motivated inflationary models such as DBI inflation [6], the size of the bispectrum and trispectrum can both in principle reach the observable limit of future experiments. Therefore it is crucial to study the shapes of higher-point functions in such inflationary models.

The CMB bispectrum for single field inflation has been studied intensively, see, e.g., [7-11]. The four-point function for slow-roll inflation, however, has only recently been studied [12], where it was found that the inflationary trispectrum generated by slow-roll models is too small to be observed even by future experiments. In this note we compute the shape of the trispectrum for DBI inflationary models and more generally a class of single field models considered in [13] where the inflationary Lagrangian is a general function of the inflaton and its first derivative. We find that for $n$-point functions, there are $n-1$ independent shapes that could be potentially large, providing useful information in distinguishing various inflation models. In the context of slow-roll inflation, Maldacena [7] has proposed an interesting consistency relation for the three-point function in a squeezed limit where one of the momentum modes has a much longer wavelength than the other modes. The importance of this relation is that its violation, if observed experimentally, could rule out a large class of inflationary scenarios model-independently [14]. In view of this, we derive a set of consistency conditions for higherpoint functions, generalizing Maldacena's result. Although some aspects of the consistency conditions for the trispectrum in slow-roll models have recently been discussed in [12], our result is applicable to a more general inflationary Lagrangian and to cases when there are more than one long wavelength modes. We show that the shapes of the trispectrum we found satisfy these generalized consistency conditions. We end with some final remarks and discuss the implications for future CMB experiments.

Let us begin with DBI inflation which was proposed in [6] and studied subsequently in e.g. [9,15-17]. Although DBI inflation can be formulated without referring to branes and extra dimensions, it is most easily visualized as describing a $D$-brane moving relativistically in a warped throat where the inflaton $\phi$ is the position of the $D$-brane. For a warp factor $f(\phi)$ and an inflaton potential $V(\phi)$, the inflationary Lagrangian is

$$
\begin{aligned}
S= & \frac{M_{\mathrm{Pl}}^{2}}{2} \int d^{4} x \sqrt{-g} R-\int d^{4} x \sqrt{-g}\left[f(\phi)^{-1}\right. \\
& \times \sqrt{1-f(\phi)\left(\dot{\phi}^{2}-a(t)^{-2}(\nabla \phi)^{2}\right)} \\
& \left.-f(\phi)^{-1}+V(\phi)\right]
\end{aligned}
$$

where $a(t)=e^{H t}$ is the scale factor and $H=\frac{\dot{a}(t)}{a(t)}$ is the Hubble parameter in the Friedmann-Robertson-Walker (FRW) universe. The background solution for the inflaton $\phi$ is spatially homogeneous. The sound speed, which 
describes the speed of fluctuations on this background, is defined as

$$
c_{s} \equiv \sqrt{1-f(\phi) \dot{\phi}^{2}}
$$

If the potential $V(\phi)$ is not flat, the inflaton will quickly approach the relativistic speed limit $\dot{\phi}^{2} \sim f(\phi)^{-1}$ and so $c_{s} \ll 1$. We will see that for DBI inflation, the bispectrum and trispectrum scale with the sound speed as:

$$
f_{N L} \sim \frac{1}{c_{s}^{2}}, \quad \tau_{N L} \sim \frac{1}{c_{s}^{4}},
$$

This can be easily understood as follows. By expanding the DBI Lagrangian in the small sound speed limit, we find that the $n$-point function scales like $\frac{1}{c_{s}^{3 n-5}}$. Since $f_{N L}$ is proportional to the three-point function divided by the square of the power spectrum, it scales as $\frac{1}{c_{s}^{2}}$. Similarly, $\tau_{N L}$ is proportional to the four-point function divided by the cube of the power spectrum, and hence it scales as $\frac{1}{c_{s}^{4}}$. Thus, there can exist models with a sufficiently small sound speed $c_{s}$ that the size of the trispectrm $\tau_{N L}$ is bigger than the sensitivity level of future experiments $\tau_{N L} \geq 560$, but yet not too small to be already ruled out by the WMAP bound on the CMB bispectrum $f_{N L}$. As analogous to the bispectrum, we expect the bound on $\tau_{N L}$ to depend on its shape [18] and so it would be interesting to carry out a similar analysis for the trispectrum. We note, however, that the bound on the trispectrum is rather weak and so only in the small sound speed limit and the leading contributions to $\tau_{N L}$ could possibly be observable in future experiments. In the small sound speed limit, the non-Gaussianities are dominated by the inflaton's derivative interactions. The contributions from the gravity sector and potential terms of the scalar are suppressed by slow-roll parameters, so they are unobservably small and we will not consider these subleading contributions in this paper. However, it is of theoretical interests [12] to study in more details these subleading contributions since they may provide a testing ground for theoretical issues such as the generalized Maldacena's consistency conditions that we will present here.

To compute the leading shape of the four-point function, we consider a perturbation $\alpha=\delta \phi$ around the background solution, and only keep the leading terms in the small sound speed limit, which come from expanding the square root term in the DBI action. The action up to quartic order is:

$$
\begin{gathered}
\mathcal{L}_{2}=\frac{a^{3}}{2 c_{s}^{3}}\left[\dot{\alpha}^{2}-a^{-2} c_{s}^{2}(\nabla \alpha)^{2}\right] \\
\mathcal{L}_{3}=\frac{a^{3}}{2 c_{s}^{5} \dot{\phi}}\left[\dot{\alpha}^{3}-a^{-2} c_{s}^{2} \dot{\alpha}(\nabla \alpha)^{2}\right]
\end{gathered}
$$

$$
\begin{aligned}
\mathcal{L}_{4}= & \frac{a^{3}}{8 c_{s}^{7} \dot{\phi}^{2}}\left[5 \dot{\alpha}^{4}-6 a^{-2} c_{s}^{2} \dot{\alpha}^{2}(\nabla \alpha)^{2}\right. \\
& \left.+a^{-4} c_{s}^{4}(\nabla \alpha)^{2}(\nabla \alpha)^{2}\right] .
\end{aligned}
$$

From the quadratic term we can solve for the perturbation and quantize it according to the standard procedures of quantum field theory:

$$
\begin{aligned}
\alpha(t, \mathbf{x})= & \frac{1}{(2 \pi)^{3}} \int d^{3} k[u(\tau, \mathbf{k}) a(\mathbf{k}) \\
& \left.+u^{*}(\tau,-\mathbf{k}) a^{\dagger}(-\mathbf{k})\right] e^{i \mathbf{k} \cdot \mathbf{x}},
\end{aligned}
$$

where $u(\tau, \mathbf{k})=\frac{H}{\sqrt{2 k^{3}}}\left(1+i k c_{s} \tau\right) e^{-i k c_{s} \tau}$ is the solution of the quadratic Lagrangian, and $\tau=-\frac{1}{a H}$ is the conformal time. The creation and annihilation operators satisfy the usual commutation relation $\left[a(\mathbf{k}), a^{\dagger}\left(\mathbf{k}^{\prime}\right)\right]=(2 \pi)^{3} \delta^{3}(\mathbf{k}-$ $\left.\mathbf{k}^{\prime}\right)$.

In the small sound speed limit, the primordial density perturbation $\zeta$ is dominated by the fluctuation of the inflaton $\alpha$, and is simply related to it by $\zeta=\frac{H}{\dot{\phi}} \alpha$ [6]. The two-point function is

$$
\left\langle\zeta\left(\mathbf{k}_{1}\right) \zeta\left(\mathbf{k}_{2}\right)\right\rangle=(2 \pi)^{3} \delta^{3}\left(\mathbf{k}_{1}+\mathbf{k}_{2}\right) \frac{P_{k_{1}}^{\zeta}}{2 k_{1}^{3}},
$$

where $P_{k}^{\zeta}=\frac{H^{4}}{\bar{\phi}^{2}}$ is the power spectrum.

We can compute the four-point function from the quartic term (6) in DBI inflation

$$
\begin{aligned}
\left\langle\zeta\left(\mathbf{k}_{1}\right) \zeta\left(\mathbf{k}_{2}\right) \zeta\left(\mathbf{k}_{3}\right) \zeta\left(\mathbf{k}_{4}\right)\right\rangle= & (2 \pi)^{3} \delta^{3}\left(\mathbf{k}_{1}+\mathbf{k}_{2}+\mathbf{k}_{3}+\mathbf{k}_{4}\right) \\
& \times\left(\frac{H^{2}}{\dot{\phi}}\right)^{6} \frac{1}{c_{s}^{4}} \frac{1}{\prod_{i=1}^{4} k_{i}^{3}} \\
& \times\left(A_{1}+A_{2}+A_{3}\right)
\end{aligned}
$$

where $A_{1}, A_{2}, A_{3}$ are contributions from the three terms in (6):

$$
\begin{aligned}
A_{1}=45 \frac{\prod_{i} k_{i}^{2}}{K^{5}} & \\
A_{2}= & -\frac{3}{16} \frac{k_{1}^{2} k_{2}^{2}\left(\mathbf{k}_{3} \cdot \mathbf{k}_{4}\right)}{K^{3}}\left(1+\frac{3\left(k_{3}+k_{4}\right)}{K}+\frac{12 k_{3} k_{4}}{K^{2}}\right) \\
& + \text { perm. } \\
A_{3}= & \frac{1}{32} \frac{\left(\mathbf{k}_{1} \cdot \mathbf{k}_{2}\right)\left(\mathbf{k}_{3} \cdot \mathbf{k}_{4}\right)}{K}\left(1+\frac{\sum_{i<j} k_{i} k_{j}}{K^{2}}\right. \\
& \left.+\frac{3 k_{1} k_{2} k_{3} k_{4}}{K^{3}}\left(\sum_{i} \frac{1}{k_{i}}\right)+12 \frac{k_{1} k_{2} k_{3} k_{4}}{K^{4}}\right)+ \text { perm. }
\end{aligned}
$$

Here, $K=\sum_{i=1}^{4} k_{i}$ and "perm." refers to the 24 permutations of the four momenta. Momentum conservation implies that the momenta $k_{i}$ where $i=1 \ldots 4$ form a quadrilateral. The quadrilateral may be skew, i.e. not lying on a plane. For convenience we will just refer to it as a 
quadrilateral. This is in contrast to the bispectrum where the momenta form a triangle.

In order to compare with the value of $\tau_{N L}$ from experiments, we need to specify a configuration of the quadrilateral to evaluate the four-point function. We consider the "equilateral configuration" in [12], where the 4 momenta have the same amplitudes $k$, and hence the angles between them satisfy $\cos \left(\theta_{12}\right)=\cos \left(\theta_{34}\right), \quad \cos \left(\theta_{13}\right)=\cos \left(\theta_{24}\right)$, $\cos \left(\theta_{14}\right)=\cos \left(\theta_{23}\right)$, and $\sum_{m=1}^{3} \cos \left(\theta_{m 4}\right)=-1$. Here $\theta_{i j}$ is the angle between $\mathbf{k}_{i}$ and $\mathbf{k}_{j}$. Using the definition of $\tau_{N L}$, we find that for DBI inflation

$$
\begin{aligned}
\tau_{N L}= & \frac{2^{3 / 2}}{c_{s}^{4}\left(\sum_{m=1}^{3}\left(1+\cos \left(\theta_{m 4}\right)\right)^{-(3 / 2)}\right.}\left[\frac{45}{1024}-\frac{39}{512}\right. \\
& \left.\times \sum_{m=1}^{3} \cos \left(\theta_{m 4}\right)+\frac{25}{256} \sum_{m=1}^{3} \cos ^{2}\left(\theta_{m 4}\right)\right]
\end{aligned}
$$

For a generic angular configuration, we can roughly estimate that $\tau_{N L} \sim \frac{0.1}{c_{s}^{4}}$. This confirms our claim that there are models that give rise to observable trispectrum, but are not ruled out by current bounds on the bispectrum, e.g., when $c_{s} \sim 0.1$.

It is straightforward to generalize the analysis to a class of models where the inflation Lagrangian is a general function $P(X, \phi)$, with $X=\frac{1}{2} g^{\mu \nu} \partial_{\mu} \phi \partial_{\nu} \phi[13]$. The sound speed is defined as

$$
c_{s}^{2}=\frac{P_{, X}}{P_{, X}+2 X P_{, X X}} .
$$

Again we consider only the leading contribution in the small sound speed limit. In this case the derivative of the Lagrangian with $\phi$, i.e., $P_{, \phi}$, does not give rise to shapes large enough to be observed. One can show that the leading quartic term is

$$
\begin{aligned}
\mathcal{L}_{4}= & a^{3}\left[\frac{1}{24} \dot{\phi}^{4} \frac{\partial^{4} P}{\partial X^{4}} \dot{\alpha}^{4}+\frac{1}{4} \dot{\phi}^{2} \frac{\partial^{3} P}{\partial X^{3}} \dot{\alpha}^{2}\left(\dot{\alpha}^{2}-a^{-2}(\nabla \alpha)^{2}\right)\right. \\
& \left.+\frac{1}{8} \frac{\partial^{2} P}{\partial X^{2}}\left(\dot{\alpha}^{2}-a^{-2}(\nabla \alpha)^{2}\right)^{2}\right]
\end{aligned}
$$

So we found 3 independent shapes (i.e., momentum dependences). A convenient basis is the set of shapes $A_{1}, A_{2}$, $A_{3}$ we found earlier.

The shapes of the four-point function $A_{1}, A_{2}, A_{3}$ can be used to distinguish between models with different parameters $\frac{\partial^{4} P}{\partial X^{4}}, \frac{\partial^{3} P}{\partial X^{3}}, \frac{\partial^{2} P}{\partial X^{2}}$. To illustrate this, we can compare the situation with that of the bispectrum, where the shapes were computed in $[9,11]$. There it was found that at the leading order there are two large shapes of nonGaussianities, denoted by $A_{\lambda}$ and $A_{c}$ :

$$
\begin{aligned}
& A_{\lambda} \sim \frac{k_{1}^{2} k_{2}^{2} k_{3}^{2}}{K^{3}}, \\
& A_{c} \sim-\frac{1}{K} \sum_{i>j} k_{i}^{2} k_{j}^{2}+\frac{1}{2 K^{2}} \sum_{i \neq j} k_{i}^{2} k_{j}^{3}+\frac{1}{8} \sum_{i} k_{i}^{3} .
\end{aligned}
$$

However, these two shapes are functions of the momentum triangle and look qualitatively similar in plots [9]. In particular, in the squeezed limit $k_{1} \rightarrow 0$, one can easily show these two shapes have the same asymptotic behaviors $A_{\lambda}, \quad A_{c} \sim k_{1}^{2}$. The shapes of the four-point function Eqs. (10)-(12) are more distinguishable. For example, we can take a squeezed limit $k_{1} \rightarrow 0$, and the remaining three sides of the quadrilateral form a triangle. The asymptotic behaviors of the shapes are

$$
\begin{aligned}
A_{1} \sim & k_{1}^{2}\left(\frac{k_{2}^{2} k_{3}^{2} k_{4}^{2}}{K^{5}}\right) \\
A_{2} \sim & \frac{k_{1} k_{2} k_{3} k_{4}}{K^{3}}\left[k_{3} k_{4} \cos \left(\theta_{12}\right)\left(1+\frac{3 k_{2}}{K}\right)+k_{2} k_{4} \cos \left(\theta_{13}\right)\right. \\
& \left.\times\left(1+\frac{3 k_{3}}{K}\right)+k_{2} k_{3} \cos \left(\theta_{14}\right)\left(1+\frac{3 k_{4}}{K}\right)\right] \\
A_{3} \sim & \frac{k_{1} k_{2} k_{3} k_{4}}{K}\left(1+\frac{k_{2} k_{2}+k_{2} k_{4}+k_{3} k_{4}}{K^{2}}+\frac{3 k_{2} k_{3} k_{4}}{K^{3}}\right) \\
& \times\left[\cos \left(\theta_{12}\right) \cos \left(\theta_{34}\right)+\cos \left(\theta_{13}\right) \cos \left(\theta_{24}\right)\right. \\
& \left.+\cos \left(\theta_{14}\right) \cos \left(\theta_{23}\right)\right]
\end{aligned}
$$

In the squeezed limit of a generic configuration of the quadrilateral, the first shape scales as $A_{1} \sim k_{1}^{2}$, and two other shapes scale as $A_{2}, A_{3} \sim k_{1}$. So this in principle provides a more refined discriminator between different inflation models than the bispectrum. Another interesting configuration is when $\mathbf{k}_{1}, \mathbf{k}_{2}$ and $\mathbf{k}_{3}$ are orthogonal to each other and $\mathbf{k}_{4}=-\mathbf{k}_{1}-\mathbf{k}_{2}-\mathbf{k}_{3}$. In this configuration, the shape $A_{3}$ vanishes. In particular, this provides a distinction between DBI inflation and the power law $k$-inflation model in [19]. The simplest power law $k$-inflation has a Lagrangian $P(X, \phi) \sim\left(-X+X^{2}\right) / \phi^{2}$. It was shown in $[13,19]$ that the model has a power law inflationary solution and the inflaton moves at constant speed. In the small sound speed limit, the power law $k$-inflation model has $\tau_{N L} \sim \frac{1}{c_{s}^{4}}$ and the shape of the 4-point function is dominated by the shape $A_{3}$, so it vanishes in the configuration mentioned above and this feature distinguishes it from DBI inflation.

One can go to more complicated corners of the configuration space of the quadrilateral and study the different behaviors of the shapes $A_{1}, A_{2}, A_{3}$, but it should be clear that the configuration space of a quadrilateral is much bigger than that of a triangle, and thus provides a more powerful discriminator for inflationary models.

Now we derive a generalized consistency condition for $n$-point function, and test it in various models for which we have computed the four-point function. In single field inflation, the density perturbation is generated by a scalar 
perturbation $\zeta$ whose $l$-point function is:

$$
\begin{aligned}
\left\langle\zeta\left(\mathbf{k}_{1}\right) \cdots \zeta\left(\mathbf{k}_{l}\right)\right\rangle= & (2 \pi)^{3} \delta^{3}\left(\mathbf{k}_{1}+\cdots+\mathbf{k}_{l}\right) \\
& \times P^{l}\left(\mathbf{k}_{1}, \cdots, \mathbf{k}_{l}\right)
\end{aligned}
$$

The scalar perturbation $\zeta$ has scaling behavior of degree -3 , so the $l$-point function $P^{l}\left(\mathbf{k}_{1}, \cdots, \mathbf{k}_{l}\right)$ is roughly a degree $3-3 l$ homogeneous function of $\mathbf{k}_{i}, i=1, \cdots, l$. We can define the spectral index of $l$-point functions analogously to that of the two-point function

$$
\begin{aligned}
n_{l}-1 & =\frac{d \log \left(P^{l}\left(\mathbf{k}_{1}, \cdots, \mathbf{k}_{l}\right)\right)}{d \log (k)}+3 l-3 \\
& =\frac{d \log \left(\left\langle\zeta\left(\mathbf{k}_{1}\right) \cdots \zeta\left(\mathbf{k}_{l}\right)\right\rangle\right)}{d \log (k)}+3 l
\end{aligned}
$$

In the above definition all the $\mathbf{k}_{i}$ are taken to be of the same order of magnitude as $k$, e.g. $\frac{d \log \left(k_{i}\right)}{d \log (k)}=1$. When $l=2$, this is just the usual spectral index of the power spectrum $n_{s}-$ 1. Another example is $l=3$. In this case the three-point spectral index is

$$
n_{3}-1=\frac{d \log \left(f_{N L}\right)}{d \log (k)}+2\left(n_{s}-1\right)
$$

where $\frac{d \log \left(f_{N L}\right)}{d \log (k)}$ is known as the running of non-Gaussianity [20]. In an inflationary model where the physical parameters vary slowly within a Hubble time, the $l$-point spectral index is very small, i.e. $\left|n_{l}-1\right| \ll 1$.

We will take the squeezed limit of the $n$-point function, where $l$ of the momenta $(2 \leq l<n)$ are much bigger than the others, i.e.,

$$
k_{1}, \cdots, k_{l} \gg k_{l+1}, \cdots, k_{n} .
$$

The long wavelength modes $k_{l+1}, \cdots, k_{n}$ exit the horizon and are frozen much earlier than the short wave modes $k_{1}, \cdots, k_{l}$. Their effect is to act as a background that scales the spatial coordinates, since they perturb the FRW metric as $d s^{2}=d t^{2}-a(t)^{2} e^{2 \zeta_{B}} d \mathbf{x}^{2}$. The $l$-point function in a background perturbation $\zeta_{B} \sim 10^{-5}$ is

$$
\left\langle\zeta\left(\mathbf{x}_{1}\right) \cdots \zeta\left(\mathbf{x}_{l}\right)\right\rangle_{B}=\left\langle\zeta\left(e^{\zeta_{B}} \mathbf{x}_{1}\right) \cdots \zeta\left(e^{\zeta_{B}} \mathbf{x}_{l}\right)\right\rangle .
$$

The background $\zeta_{B}$ is roughly constant around the short wave length scale $\left|x_{i}-x_{j}\right|$ with $1 \leq i<j \leq l$, and can be evaluated at $\mathbf{x}_{0}=\frac{\mathbf{x}_{1}+\cdots+\mathbf{x}_{l}}{l}$. We expand the above equation to first order in the background

$$
\begin{aligned}
\left\langle\zeta\left(\mathbf{x}_{1}\right) \cdots \zeta\left(\mathbf{x}_{l}\right)\right\rangle_{B}= & \left\langle\zeta\left(\mathbf{x}_{1}\right) \cdots \zeta\left(\mathbf{x}_{l}\right)\right\rangle+\zeta_{B}\left(\mathbf{x}_{0}\right)\left\{\left(\frac{d}{d \zeta_{B}}\right.\right. \\
& \left.\left.\times\left\langle\zeta\left(e^{\zeta_{B}} \mathbf{x}_{1}\right) \cdots \zeta\left(e^{\zeta_{B}} \mathbf{x}_{l}\right)\right\rangle\right)\left.\right|_{\zeta_{B}=0}\right\}
\end{aligned}
$$

The zeroth order term is independent of the background so will not contribute to the final result. After a Fourier transformation to momentum space, the first order term in the background expansion becomes

$$
\begin{aligned}
\left\langle\zeta\left(\mathbf{k}_{1}\right) \cdots \zeta\left(\mathbf{k}_{l}\right)\right\rangle_{B}= & \int d^{3} \mathbf{k}_{B} \zeta_{B}\left(\mathbf{k}_{B}\right) \\
& \times\left\{\frac { d } { d \zeta _ { B } } \left(e ^ { - 3 l \zeta _ { B } } \left\langle\zeta\left(e^{-\zeta_{B}}\left(\mathbf{k}_{1}-\frac{\mathbf{k}_{B}}{l}\right)\right) \cdots\right.\right.\right. \\
& \left.\left.\left.\times \zeta\left(e^{-\zeta_{B}}\left(\mathbf{k}_{l}-\frac{\mathbf{k}_{B}}{l}\right)\right)\right\rangle\right)\left.\right|_{\zeta_{B}=0}\right\}
\end{aligned}
$$

Here we define $\mathbf{k}_{B} \equiv \mathbf{k}_{1}+\cdots+\mathbf{k}_{l}$, which satisfies $k_{B} \ll$ $k_{i}, i=1, \cdots l$. At the leading order of the squeezed limit we can neglect the background momentum in the $l$-point function, and use our definition of the $l$-point spectral index to find

$$
\begin{aligned}
\left\langle\zeta\left(\mathbf{k}_{1}\right) \cdots \zeta\left(\mathbf{k}_{l}\right)\right\rangle_{B}= & -(2 \pi)^{3}\left(n_{l}-1\right) P^{l}\left(\mathbf{k}_{1}, \cdots, \mathbf{k}_{l}\right) \\
& \times \zeta\left(\mathbf{k}_{1}+\cdots+\mathbf{k}_{l}\right)
\end{aligned}
$$

Plugging in the expression for the $n$-point function, we find a generalization of Maldacena's consistency condition to $n$-point function in the squeezed limit $k_{1}, \cdots, k_{l} \gg$ $k_{l+1}, \cdots, k_{n}$ :

$$
\begin{aligned}
P^{n}\left(\mathbf{k}_{1}, \cdots, \mathbf{k}_{n}\right)= & -(2 \pi)^{3}\left(n_{l}-1\right) P^{l}\left(\mathbf{k}_{1}, \cdots, \mathbf{k}_{l}\right) \\
& \times P^{n-l+1}\left(\mathbf{k}_{1}+\cdots \mathbf{k}_{l}, \mathbf{k}_{l+1}, \cdots, \mathbf{k}_{n}\right)
\end{aligned}
$$

In the case of DBI inflation, the $n$-point function on the left hand side of the consistency condition (26) scales as $\frac{\left(P_{k}^{\zeta}\right)^{n-1}}{c_{s}^{2 n-4}}$, while the right hand side scales as $\frac{\left(P_{k}^{\zeta}\right)^{n-1}}{c_{s}^{2 n-6}}$, so the $n$-point function must vanish in the squeezed limits at leading order for small sound speed. We see indeed the shapes $A_{1}, A_{2}$ and $A_{3}$ in (10)-(12) all vanish in a squeezed limit when there is a long wave mode $k_{i} \rightarrow 0$, in agreement with the $n$-point consistency condition (26). A more nontrivial check of (26) is when $P^{n}\left(\mathbf{k}_{1}, \cdots, \mathbf{k}_{n}\right)$ is nonvanishing in the squeezed limit, e.g., one may check the validity of (26) using the recent results for slow-roll inflation [12] (together with subleading terms which are yet to be determined). Besides serving as a check on the calculations, the generalized consistency condition (26) if violated can, in the spirit of [14], rule out a large class of models experimentally.

For a general Lagrangian $P(X, \phi)$, the $n$-point function has $n-1$ independent shapes proportional to the derivatives of the Lagrangian $\frac{\partial^{i} P}{\partial X^{i}}$, where $i=2,3, \cdots, n$. It is not difficult to compute the shapes and show that they vanish in the squeezed limit, though it is difficult to measure such higher-point functions experimentally.

We emphasize that the CMB trispectrum, athough difficult to observe, could provide a useful discriminator for inflationary models. In particular, if large nonGaussianities are observed experimentally in the future, it may still be difficult to distinguish via the bispectrum DBI inflation from other inflationary models governed by derivative interactions, e.g., the models in [11]. The trispec- 
INFLATIONARY TRISPECTRUM FOR MODELS WITH ...

trum we computed may serve as an extra filter to zero-in on string inflationary scenarios.

We thank X. Chen, D. Chung, G. Geshnizjani, S. Kachru, B. Underwood for discussions. This work was
PHYSICAL REVIEW D 74, 121301(R) (2006)

supported in part by NSF CAREER Grant No. PHY0348093, DOE grant No. DE-FG-02-95ER40896, a Research Innovation Grant and a Cottrell Scholar Grant from Research Corporation.
[1] D. N. Spergel et al., astro-ph/0603449.

[2] L. Alabidi and D. H. Lyth, J. Cosmol. Astropart. Phys. 05 (2006) 016.

[3] N. Kogo and E. Komatsu, Phys. Rev. D 73, 083007 (2006).

[4] P. Creminelli, L. Senatore, and M. Zaldarriaga, astro-ph/ 0606001.

[5] T. Okamoto and W. Hu, Phys. Rev. D 66, 063008 (2002).

[6] E. Silverstein and D. Tong, Phys. Rev. D 70, 103505 (2004); M. Alishahiha, E. Silverstein, and D. Tong, Phys. Rev. D 70, 123505 (2004).

[7] J. M. Maldacena, J. High Energy Phys. 05 (2003) 013.

[8] V. Acquaviva, N. Bartolo, S. Matarrese, and A. Riotto, Nucl. Phys. B667, 119 (2003).

[9] X. Chen, M.x. Huang, S. Kachru, and G. Shiu, hep-th/ 0605045 .

[10] D. Seery and J. E. Lidsey, J. Cosmol. Astropart. Phys. 06 (2005) 003; J. Cosmol. Astropart. Phys. 09 (2005) 011.

[11] A. Gruzinov, Phys. Rev. D 71, 027301 (2005).
[12] D. Seery, J. E. Lidsey, and M. S. Sloth, astro-ph/0610210.

[13] J. Garriga and V.F. Mukhanov, Phys. Lett. B 458, 219 (1999).

[14] P. Creminelli and M. Zaldarriaga, J. Cosmol. Astropart. Phys. 10 (2004) 006.

[15] X. Chen, Phys. Rev. D 71, 063506 (2005); J. High Energy Phys. 08 (2005) 045.

[16] G. Shiu and B. Underwood, hep-th/0610151; S. Kecskemeti, J. Maiden, G. Shiu, and B. Underwood, J. High Energy Phys. 09 (2006) 076.

[17] S. E. Shandera and S. H. Tye, J. Cosmol. Astropart. Phys. 05 (2006) 007.

[18] D. Babich, P. Creminelli, and M. Zaldarriaga, J. Cosmol. Astropart. Phys. 08 (2004) 009.

[19] C. Armendariz-Picon, T. Damour, and V.F. Mukhanov, Phys. Lett. B 458, 209 (1999).

[20] X. Chen, Phys. Rev. D 72, 123518 (2005). 